\title{
AFECTACIONES PSICOLÓGICAS DE NIÑOS Y ADOLESCENTES EXPUESTOS AL CONFLICTO ARMADO EN UNA ZONA RURAL DE COLOMBIA ${ }^{1}$
}

\author{
NOHELIA HEWITT RAMÍREZ*, CARLOS ANDRÉS GANTIVA DÍAZ, ANDERSSEN VERA MALDONADO, \\ MÓNICA PAULINA CUERVO RODRÍGUEZ, NELLY LILIAM HERNÁNDEZ OLAYA \\ UNIVERSIDAD DE SAN BUENAVENTURA, BOGOTÁ - COLOMBIA \\ FERNANDO JUÁREZ \\ UNIVERSIDAD DEL ROSARIO, BOGOTÁ - COLOMBIA \\ ARTURO JOSÉ PARADA BAÑOS \\ CORPORACIÓN IMPACTO VITAL, BOGOTÁ - COLOMBIA
}

Recibido, noviembre 28/2013

Concepto evaluación, diciembre 15/2013

Aceptado, diciembre 27/2013
Referencia: Hewitt, N., Gantiva, C.A., Vera, A., Cuervo, M.P., Hernández, N.L., Juárez, F. \& Parada, A. J. (2014). Afectaciones psicológicas de niños y adolescentes expuestos al conflicto armado en una zona rural de Colombia. Acta Colombiana de Psicología, 17(1), 79-89. doi: 10.14718/ACP.2014.17.1.9

Resumen

Se determinaron las afectaciones psicológicas de 284 niños y adolescentes expuestos al conflicto armado en una zona rural colombiana, seleccionados mediante un muestreo aleatorio por afijación proporcional. Los instrumentos aplicados fueron: la Lista de chequeo de comportamiento infantil, el Auto-reporte de comportamientos de jóvenes, la Lista de síntomas postraumáticos, la Escala de estrategias de afrontamiento para adolescentes y la Escala de resiliencia para escolares. El $72 \%$ de la población presentó afectaciones psicológicas: el 64.4\%, conductas internalizadas, el 47\%, conductas externalizadas en rango clínico. El 32\%, problemas somáticos; el 56\%, se encontraba en riesgo de estrés postraumático, y el 93\% consumía alcohol en grado moderado. La estrategia de afrontamiento más utilizada era dejar que las cosas se arreglaran solas. Se encontró una alta necesidad de atención en salud. Ser hombre constituyó un factor de riesgo de depresión, agresión y problemas sociales en los niños. A su vez, tener hasta doce años y estar cursando un grado escolar bajo, lo fue para los síntomas somáticos en adolescentes. Los resultados evidenciaron la afectación en la salud mental de los participantes.

Palabras clave: niños, adolescentes, conductas internalizadas, conductas externalizadas, factores de riesgo.

\section{PSYCHOLOGICAL EFFECTS ON CHILDREN AND ADOLESCENTS EXPOSED TO ARMED CONFLICT IN A RURAL AREA OF COLOMBIA}

\begin{abstract}
Psychological effects were determined in 284 children and adolescents exposed to armed conflict in a rural area of Colombia, selected through random sampling proportional allocation. The instruments applied were: The Child Behavior Checklist, the Youth Behavior Self-report, the Trauma Symptoms Checklist for Children, the Coping Scale for Adolescents and the Resilience Scale for School Children. Findings showed that $72 \%$ of the population suffers from psychological problems, 64.4 from internalizing behavior and $47 \%$ from externalizing behavior within clinical range. It was also found that $32 \%$ had somatic problems, $56 \%$ were at risk for PTSD and 93\% had moderate alcohol consumption. The most frequently used coping strategy was to leave things get fixed by themselves. There was a high need for health care. Being male constituted a risk factor for depression, aggression and social problems in children. In turn, being up to12 years old and a student of a lower grade were risk factors for somatic symptoms in adolescents. Results clearly revealed the impact on mental health of the population under study.

Key words: children, adolescents, internalizing behaviors, externalizing behaviors, risk factors
\end{abstract}

\footnotetext{
* Magíster en Psicología. Grupo de investigación Avances en psicología clínica. Carrera 8H No. 172-20 Bogotá - Facultad de Psicología Universidad de San Buenaventura Bogotá. Código Postal 1100100. Teléfono fijo 0057-1-6671098. * nhewitt@usbbog.edu.co

1 Investigación realizada en alianza entre la Corporación Impacto Vital, la Facultad de Psicología de la Universidad de San Buenaventura, sede Bogotá y con el financiamiento de la Agencia de los Estados Unidos para el Desarrollo Internacional (USAID). Agradecemos a las madres y padres, niños (as), a los jóvenes participantes y a la Alcaldía de Viotá, Cundinamarca, Colombia.
} 


\title{
AFETAÇÓES PSICOLÓGICAS DE CRIANÇAS E ADOLESCENTES EXPOSTOS AO CONFLITO ARMADO EM UMA ZONA RURAL DA COLÔMBIA
}

Resumo

\begin{abstract}
Determinaram-se as afetações psicológicas de 284 crianças e adolescentes expostos ao conflito armado em uma zona rural colombiana, selecionados mediante uma amostragem aleatória. Os instrumentos aplicados foram: a Lista de checagem de comportamento infantil, a autoavaliação de comportamentos de jovens, a Lista de sintomas pós-traumáticos, a Escala de estratégias de enfrentamento para adolescentes e a Escala de resiliência para escolares. $72 \%$ da população apresentou afetações psicológicas: $64.4 \%$, condutas internalizadas, $47 \%$, condutas externalizadas. $32 \%$, problemas somáticos; $56 \%$, se encontrava em risco de estresse pós-traumático, e 93\% consumia álcool de forma moderada. A estratégia de enfretamento mais utilizada era deixar que as coisas se resolvessem sozinhas. Foi encontrada uma alta necessidade de atenção em saúde. Ser homem constituiu um fator de risco de depressão, agressão e problemas sociais. Ao mesmo tempo que ter até doze anos e estar cursando uma série escolar baixa, foi para os sintomas somáticos em adolescentes. Os resultados evidenciaram a afetação da saúde mental dos participantes.

Palavras chave: crianças, adolescentes, condutas internalizadas, condutas externalizadas, fatores de risco.
\end{abstract}

La guerra destruye comunidades, familias e irrumpe en el desarrollo social y económico de las naciones (Bell, Méndez, Martínez, Palma, \& Bosch, 2012). Miles de niños y jóvenes han muerto, y otros han sido expuestos a situaciones complejas como el secuestro, la pérdida de los padres y el desplazamiento, entre otras, que han atentado contra su seguridad, estabilidad mental y emocional (Al-Obaidi, Budosan \& Jefrey, 2010; Betancourt, McBain, Newnham \& Brennan, 2012; Mels, 2012).

Los efectos de la guerra abarcan deficiencias físicas y psicológicas que perjudican a niños y adultos (Miller \& Rasmussen, 2010; Murthy \& Lakshminarayana, 2006), afectan seriamente la vida social, la salud mental, la salud integral y el bienestar; incluyen las redes de atención familiar y comunitaria; interfieren en el acceso a las necesidades básicas, la educación y la espiritualidad, e incrementan las tasas de prevalencia de los trastornos mentales, incluyendo los del estado de ánimo, de ansiedad, y los trastornos de conducta (Betancourt et al., 2012; Haroz, Murray, Bolton, Betancourt, \& Bass, 2013; Miller \& Rasmussen, 2010; Tol, Song \& Jordans, 2013).

La Organización Mundial de la Salud (OMS) (2012) estima que en las situaciones de conflicto armado en todo el mundo, el 10\% de las personas que experimenta acontecimientos traumáticos tendrá graves problemas de salud mental, y otro $10 \%$ desarrollará comportamientos que obstaculizan su capacidad de funcionar eficazmente. Las condiciones más comunes son la depresión, la ansiedad y los problemas psicosomáticos tales como insomnio, dolor de espalda y dolores de estómago (Londoño et al, 2008; Bell et al., 2012; Betancourt et al., 2012).

Esta problemática social afecta las cuatro vías principales en la salud mental y física de los niños y adolescentes, como son el conflicto impulsado por el desplazamiento que aumenta la mortalidad infantil, la inseguridad alimentaria y de desnutrición; el riesgo de violencia sexual por parte de los actores armados y la discapacidad física y psicológica, especialmente entre los inmersos en el conflicto (Betancourt et al., 2012; Betancourt et al., 2013; Tamashiro, 2010).

Se ha documentado que la exposición a eventos traumáticos relacionados con la guerra contribuye a desórdenes en la salud mental que pueden perdurar a lo largo del desarrollo de los niños y adolescentes (Betancourt \& Khan, 2008; Comer \& Kendall, 2007; Fazel, Reed, Panter-Brick, \& Stein, 2012; López-Navarrete et al., 2007; Masten \& Narayan, 2012; Neuner et al., 2008). Los niños y niñas son particularmente vulnerables a la violencia y a los efectos derivados de ésta (Haroz et al., 2013), dado su estatus único de individuo en proceso de crecimiento y desarrollo cuyas estructuras básicas de la personalidad y su visión del mundo están construyéndose (Pereda, 2012).

Entre las afectaciones psicológicas que presentan los niños y adolescentes como producto de la violencia se encuentran las dificultades en el desarrollo (Mels, 2012; Murthy \& Lakshminarayana, 2006), problemas de atención, problemas cognitivos con ideas distorsionadas sobre lo ocurrido, culpa, vergüenza, inseguridad, indefensión, agresividad, pérdida de relaciones interpersonales o pérdida de confianza en el futuro, desarrollo de conductas regresivas, angustia por la separación de sus padres, miedo, ansiedad, depresión, trastornos del sueño, y labilidad emocional, entre otros, las que dependen de la etapa de desarrollo y del tipo, grado e intensidad de los hechos victimizantes (Cohen \& Mannarino, 2008; López-Navarrete et al., 2007; Pereda 2012).

El trastorno por estrés postraumático es una de estas consecuencias psicológicas, frecuentemente estudiada, en 
menores víctimas de las atrocidades de la guerra (Haroz et al., 2013; Hobfoll, Mancini, Hall, Canetti \& Bonanno, 2011; Mels, Derluyn, Broekaert \& Rosseel, 2009; Rieder \& Choonara, 2012; Yule, Dyregrov, Raundalen \& Smith, 2013; Ziaian, De Anstiss, Antoniou, Baghurst \& Sawyer, 2012), de la violencia interpersonal en general y de los atentados terroristas en particular (Betancourt et al., 2013; Hasanovic, 2011; Tol et al., 2012).

Ser testigo o víctima de violencia se ha vinculado con problemas de conducta de tipo internalizado, evidenciados más en las niñas, tales como depresión, ansiedad y baja autoestima. Se ha encontrado que la violación es el predictor más fuerte de estos problemas. Por el contrario, las conductas externalizadas como ruptura de normas, agresión y consumo de drogas se evidencian más en los niños, acompañadas de problemas interpersonales y un bajo rendimiento escolar. Es el asesinato o las lesiones a otros las que se vinculan más estrechamente con los problemas externalizados (Betancourt et al., 2012; Haroz et al., 2013; Mels, 2012; Posada, Giléde \& Parales, 2011).

Varios estudios han puesto de relieve la resiliencia en niños y adolescentes en zonas de conflicto armado. Mientras que un gran número de niños/as y adolescentes sufren síntomas de desajuste psicológico debido a la guerra, muchos de ellos se recuperan de manera natural o logran seguir funcionando social y académicamente, a pesar de la presencia de algunos síntomas (Betancourt \& Khan, 2008; Feldman \& Vengrober, 2011; Fernando \& Ferrari, 2011; Haroz et al., 2013; Mels, 2012; Pine \& Cohen, 2002; Ziaian et al., 2012).

En Colombia ha existido el conflicto armado interno en el último medio siglo (Bastidas \& Rodríguez, 2010; Sánchez et al., 2009) como consecuencia de los problemas de inestabilidad e ilegitimidad política, impunidad y desigualdad económica y social (Alzate, 2012). En las dos últimas décadas éste no sólo se incrementó, sino que se extendió en el país, de tal manera que el riesgo de morir o enfermar como víctima de la guerra aumentó, y Colombia se convirtió en uno de los países con mayor presencia de actos violentos (Comer \& Kendall, 2007; Pérez-Olmos, Fernández-Piñeres \& Rodado-Fuentes, 2005).

En un estudio realizado en tres municipios del departamento de Cundinamarca - Colombia, para determinar la prevalencia del trastorno por estrés postraumático (TEPT) infantil y factores asociados, según tipo de exposición a la guerra se concluyó que los habitantes de los municipios expuestos tuvieron 19 veces mayor probabilidad de sufrir TEPT bélico que los no expuestos. La proximidad geográfica y la alteración emocional intensa durante el trauma aumentaron el riesgo (Pérez-Olmos et al., 2005).
Lo anterior muestra que el impacto psicológico del terrorismo en los menores es significativo. La afectación psicológica resultante se constituye en un problema de salud pública sobre el que existe escasa investigación en Colombia, un país afectado por la violencia durante seis décadas (Bell et al., 2012; Sánchez et al., 2009). De acuerdo con lo expuesto, este estudio pretendió determinar las afectaciones psicológicas, y evaluar la percepción y la necesidad de atención en salud mental de la población infantil y adolescente expuesta a situación de guerra en un municipio de Colombia.

\section{MÉTODO}

\section{Participantes}

La muestra estuvo constituida por 289 niños y adolescentes (48\% (136) hombres y 52\% (153) mujeres) de siete a 16 años de edad (edad promedio diez años); el $92 \%$ estudiantes, de educación básica primaria (44\%) y secundaria (56\%) y un $8 \%$ dedicados a trabajar. Los participantes siempre habían vivido en la zona de conflicto armado, el $76 \%$ bajo la protección y cuidado de ambos padres y estaban distribuidos de forma equivalente en las áreas rural y urbana.

Dichos participantes se seleccionaron mediante un muestreo polietápico, propositivo aleatorio, que implicó una muestra intencional de la población más expuesta a las situaciones de conflicto y un muestreo aleatorio por afijación proporcional por edad y localización de vivienda (urbana o rural).

\section{Instrumentos}

Encuesta de características sociodemográficas de los participantes

Permite obtener información sobre datos sociodemográficos de los participantes, sitio de vivienda, tiempo viviendo en la zona de conflicto y régimen de salud. También contiene siete preguntas que evalúan la percepción de atención en salud y tres preguntas sobre la necesidad de atención en este aspecto. Adicionalmente, identifica si ha vivido, ha sido testigo o se ha enterado de algún evento estresante en algún momento de su vida, y si el evento ha estado relacionado con el conflicto armado.

Lista de chequeo del comportamiento infantil 6-18 años (Child Behavior Checklist, [CBCL]) (Achenbach \& Rescorla, 2001).

Consta de dos partes: la primera evalúa la adaptación social y el rendimiento académico del niño; la segunda abarca 113 ítems, a través de los cuales los padres evalúan el comportamiento de los niños. Esta segunda parte 
fue la aplicada en esta investigación. La confiabilidad es de .79 para las escalas de competencias sociales; de .78 a .97 para las escalas de los comportamientos específicos; y para las escalas totales de .95 (Achenbach \& Rescorla, 2001). Esta lista se ha utilizado en estudios multiculturales y fue validada con población colombiana por Hewitt, Vera, Jaimes y Villa (2012).

Auto reporte de los comportamientos de jóvenes 11-18 años - YSR (Achenbach \& Rescorla, 2001).

Consta de dos sub-áreas: 1) 17 ítems que miden la participación del niño en hobbies, juegos, deportes, trabajos, quehaceres, amistades y actividades. 2) 112 ítems que miden las subescalas de aislamiento, quejas somáticas, ansiedad y depresión, problemas sociales, problemas de pensamiento, problemas de atención, conductas de agresión y conductas delictivas (Achenbach \& Rescorla, 2001). Tres subescalas que se refieren a las conductas de internalización (aislamiento, quejas somáticas, ansiedad y depresión), dos, a las conductas de externalización (conductas de agresión y conductas delictivas) y tres se categorizan como no internalizadas y no externalizadas (problemas sociales, problemas de pensamiento y problemas de atención). Esta última parte fue la que se aplicó en este estudio (Achenbach \& Rescorla, 2001). Esta escala ha sido utilizada en estudios multicéntricos en países de Europa, Asia, Norte del África, América del Norte y Suramérica, demostrando un buen nivel de consistencia y confiabilidad (Rescorla et al., 2012).

\section{Lista de chequeo de sintomas traumáticos para niños (TSSC)} Trauma Symptoms Checklist for Children (Briere, 1996).

Es una escala de auto-informe de 54 ítems que mide el estrés postraumático y la sintomatología psicológica relacionada. Se compone de dos escalas de validez (que indican sobre y sub-registro de los síntomas) y seis escalas clínicas (ansiedad, depresión, estrés postraumático, problemas sexuales, disociación, e ira). Al niño se le presenta una lista de los pensamientos, sentimientos y comportamientos, y se le pide que marque la frecuencia con que le sucede cada uno de ellos. Está escrito para un nivel de lectura de ocho años, y se ha normativizado para niños y niñas entre las edades de ocho y 16 años, de diferentes contextos sociales y demográficos. Es útil en la evaluación de los niños que han sufrido eventos traumáticos, incluyendo abuso físico y sexual, victimización por parte de sus compañeros, pérdidas, el ser testigo expuesto a hechos violentos y a desastres naturales (Briere, 1996). Tiene un nivel de confiabilidad de .82 a .89 para cinco de las seis escalas. La escala clínica restante, SC, cuenta con una confiabilidad de .77 (Briere, 1996). Se utilizó la versión validada en población colombiana, que presenta una confiabilidad de .73 para ansiedad; .74 para depresión; .76 para ira; .74 para estrés postraumático y .73 para disociación (Cuevas, 2006).

Escala de estrategias de afrontamiento de adolescentes KIDCOPE (Spirito, Starck \& Williams, 1988).

Es un cuestionario diseñado para evaluar el afrontamiento en niños y adolescentes. Está diseñado en dos versiones para rango de edad de siete a doce años y de 13 a 18 años (Spirito et al., 1988). Consta de diez ítems en la versión de los mayores y 15 ítems en la de los menores, que corresponden a diez categorías de estrategias de afrontamiento cognitivas y conductuales tales como distracción, aislamiento, reestructuración cognitiva, autocrítica, culpar a los demás, resolución de problemas, regulación emocional, pensamiento mágico, apoyo social y resignación. Contiene tres formatos en los que se incluye un problema reciente, un problema escolar y una situación familiar problemática. Los menores deben relatar el acontecimiento más estresante al que han tenido que hacer frente y contestar a 15 ítems indicando (sí/no) y la eficacia percibida. Tiene una confiabilidad test retest dependiente de intervalos entre .15 a 0.83 . Se utilizó la versión adaptada en población escolar colombiana (Cuevas, 2006).

Escala de resiliencia para escolares ERE (Saavedra \& Villata, 2008)

Es una escala para ser aplicada a niños de nueve a 14 años; se aplica de manera colectiva o individual, y consta de 27 ítems con cinco alternativas de respuesta. La puntuación máxima es de 135 puntos y la mínima, 27 puntos. Se utilizó la escala validada con población escolar chilena, que cuenta con una validez de .78 y una confiabilidad de Cronbach de .88 (Saavedra \& Villata, 2008).

\section{Procedimiento}

Inicialmente se llevó a cabo un mapeo situacional que permitió identificar la población expuesta al conflicto armado y llevar a cabo la selección de la muestra. Luego, se entrenó a un grupo de cuatro psicólogos y 20 estudiantes de psicología de últimos semestres de formación en la aplicación de los instrumentos definidos. Se contactaron los rectores de las cuatro instituciones educativas de la zona, para presentarles la investigación y para que invitaran a los padres de los niños y a los adolescentes a participar en la misma. Posteriormente se citó a los padres y adolescentes a una sesión grupal en la que se les informó sobre la investigación. Una vez manifestada su voluntariedad de participar, firmaron el consentimiento y diligenciaron los cuestionarios, sesión que tuvo una duración de dos horas. Finalmente, se llevó a cabo el análisis de los resultados. 


\section{RESULTADOS}

El análisis de los resultados implicó: a) El análisis descriptivo y el estudio epidemiológico de corte por ciclo vital. b) El análisis por niveles de afectación y ciclo vital en el que se revisan los niveles de afectación normal, riesgo y clínico. c) El análisis de la percepción de salud y necesidad de atención en salud y d) Los factores de riesgo relacionados con las problemáticas psicológicas.

Análisis descriptivo de la población y estudio epidemiológico de corte por ciclo vital y niveles de afectación

Este análisis muestra las características sintomáticas de la población infantil y adolescente de acuerdo con

Tabla 1

Distribución de frecuencias del CBCL (niños) y YSR (adolescentes) según clasificación en las categorías de normal, riesgo y clínico.

\begin{tabular}{|c|c|c|c|c|c|}
\hline \multirow{2}{*}{ Problema Psicológico } & \multirow{2}{*}{ Categorías } & \multicolumn{2}{|c|}{ Niños - CBCL } & \multicolumn{2}{|c|}{ Adolescentes YSR } \\
\hline & & $\mathbf{F}$ & $(\%)$ & $\mathbf{F}$ & $(\%)$ \\
\hline \multirow[t]{3}{*}{ Ansiedad } & Normal & 80 & 63 & 90 & 55,6 \\
\hline & Riesgo & 25 & 19,7 & 47 & 29 \\
\hline & Clínico & 22 & 17,3 & 25 & 15,4 \\
\hline \multirow[t]{3}{*}{ Depresión } & Normal & 76 & 59,8 & 87 & 53,7 \\
\hline & Riesgo & 23 & 18,1 & 47 & 29 \\
\hline & Clínico & 28 & 22 & 28 & 17,3 \\
\hline \multirow[t]{3}{*}{ Problemas somáticos } & Normal & 70 & 55,1 & 55 & 34 \\
\hline & Riesgo & 18 & 14,2 & 51 & 31,5 \\
\hline & Clínico & 39 & 30,7 & 56 & 34,6 \\
\hline \multirow[t]{3}{*}{ Problemas sociales } & Normal & 90 & 70,9 & 90 & 55,6 \\
\hline & Riesgo & 20 & 15,7 & 47 & 29 \\
\hline & Clínico & 17 & 13,4 & 25 & 15,4 \\
\hline \multirow[t]{3}{*}{ Problemas de pensamiento } & Normal & 85 & 66,9 & 18 & 11,1 \\
\hline & Riesgo & 14 & 11 & 40 & 24,7 \\
\hline & Clínico & 28 & 22 & 104 & 64,2 \\
\hline \multirow[t]{3}{*}{ Problemas de atención } & Normal & 101 & 79,5 & 114 & 70,4 \\
\hline & Riesgo & 15 & 11,8 & 41 & 25,3 \\
\hline & Clínico & 11 & 8,7 & 7 & 4,3 \\
\hline \multirow[t]{3}{*}{ Rompimiento de Reglas } & Normal & 96 & 75,6 & 67 & 41,4 \\
\hline & Riesgo & 5 & 3,9 & 60 & 37 \\
\hline & Clínico & 26 & 20,5 & 35 & 21,6 \\
\hline \multirow[t]{3}{*}{ Agresión } & Normal & 83 & 65,4 & 110 & 67,9 \\
\hline & Riesgo & 29 & 22,8 & 37 & 22,8 \\
\hline & Clínico & 15 & 11,8 & 15 & 9,3 \\
\hline \multirow[t]{3}{*}{ Conductas Internalizadas } & Normal & 37 & 29,1 & 25 & 15,4 \\
\hline & Riesgo & 19 & 15 & 19 & 11,7 \\
\hline & Clínico & 71 & 55,9 & 118 & 72,8 \\
\hline \multirow[t]{3}{*}{ Conductas Externalizadas } & Normal & 64 & 50,4 & 31 & 19,1 \\
\hline & Riesgo & 17 & 13,4 & 35 & 21,6 \\
\hline & Clínico & 46 & 36,2 & 96 & 59,3 \\
\hline \multirow[t]{3}{*}{ CBCL total } & Normal & 46 & 36,2 & 5 & 3,1 \\
\hline & Riesgo & 21 & 16,5 & 15 & 9,3 \\
\hline & Clínico & 60 & 47,2 & 142 & 87,7 \\
\hline
\end{tabular}


los niveles de afectación normal, riesgo y clínico (véase tabla 1). Evidencia que los 127 niños y niñas participantes presentan en el CBCL, un alto grado de conductas externalizadas e internalizadas de ansiedad y agresión. El 47\% de la población infantil se sitúa en el rango clínico en el conjunto de problemas del CBCL. El $36 \%$ presenta conductas externalizadas y el $55.9 \%$ conductas internalizadas en el rango clínico. Las sub-escalas de rompimiento de reglas, problemas de pensamiento y depresión muestran que el $20 \%$ de los niños y niñas se ubica en un rango clínico. Se observa que el $30 \%$ se encuentra en un rango clínico en la escala de problemas somáticos.

Según el YSR, de los 162 adolescentes que participaron en el estudio, el $88 \%$ presenta problemas de comportamiento, el $73 \%$ conductas internalizadas y un $59 \%$ conductas externalizadas en el rango clínico. Es de destacar que en un $64 \%$ de los adolescentes se identifican problemas de pensamiento y en un $35 \%$ problemas somáticos en el rango clínico.

Por otra parte, según los resultados arrojados por el TSSC, se evidencian diferencias en las categorías en cada uno de los aspectos de diagnóstico, y se observa que el $56 \%$ de los adolescentes está en riesgo de problemas de ansiedad y de trastorno por estrés postraumático, y el 40\%, de disociación (véase Tabla 2).

Los datos descriptivos de la sumatoria de eventos asociados al conflicto reportan un promedio de un evento ocurrido, presenciado o vivido por alguien cercano a este grupo poblacional. El 31\% ha vivido algún evento y/o le ha ocurrido a algún familiar. El 17\% ha presenciado algún evento.

Con respecto al consumo de alcohol, los resultados de los descriptivos por categorías en el CAGE, evidenciaron que el $1 \%$ de la población adolescente se encuentra en consumo perjudicial, un $6 \%$ en consumo de riesgo y un $93 \%$ en consumo moderado. Según el Kidcope, las estrategias de afrontamiento más utilizadas por el grupo de adolescentes son dejar que las cosas se arreglen solas $(92 \%)$; intentar calmarse $(90,7 \%)$; hacer algo diferente $(88,3 \%)$; intentar sentirse mejor (87\%); hacer algo para arreglar las cosas $(85,8 \%)$; desear algo $(85,8 \%)$; o bien, ver el lado bueno de las cosas $(82,7 \%)$. Todas ellas son consideradas útiles por la población (véase Tabla 3).

La escala de resiliencia muestra niveles altos, especialmente en la utilización de redes (media 38) y en el aprendizaje (media: 37$)$. Los recursos internos son elevados (media: 53) (Tabla 4).

Tabla 2

Distribución de frecuencias y comparación de las categorías según el diagnóstico en el TSSC

\begin{tabular}{|c|c|c|c|c|c|c|}
\hline Diagnóstico & Categorías & $\mathbf{F}$ & $(\%)$ & Chi-cuadrado & Gl & $\mathbf{P}$ \\
\hline \multirow[t]{3}{*}{ Ansiedad } & Normal & 48 & $(29,6)$ & 46,37 & 2 &, 00 \\
\hline & Riesgo & 92 & $(56,8)$ & & & \\
\hline & Clínico & 22 & $(13,6)$ & & & \\
\hline \multirow[t]{3}{*}{ Depresión } & Normal & 96 & $(59,3)$ & 72,14 & 2 &, 00 \\
\hline & Riesgo & 58 & $(35,8)$ & & & \\
\hline & Clínico & 8 & $(4,9)$ & & & \\
\hline \multirow[t]{2}{*}{ Ira } & Normal & 142 & $(87,7)$ & 91,87 & 1 &, 00 \\
\hline & Riesgo & 20 & $(12,3)$ & & & \\
\hline \multirow[t]{3}{*}{ Estrés post } & Normal & 64 & $(39,5)$ & 62,03 & 2 &, 00 \\
\hline & Riesgo & 89 & $(54,9)$ & & & \\
\hline & Clínico & 9 & $(5,6)$ & & & \\
\hline \multirow[t]{3}{*}{ Disociación } & Normal & 93 & $(57,4)$ & 74,48 & 2 &, 00 \\
\hline & Riesgo & 64 & $(39,5)$ & & & \\
\hline & Clínico & 5 & $(3,1)$ & & & \\
\hline \multirow[t]{3}{*}{ Disociación Abierta } & Normal & 96 & $(59,3)$ & 78,03 & 2 &, 00 \\
\hline & Riesgo & 61 & $(37,7)$ & & & \\
\hline & Clínico & 5 & $(3,1)$ & & & \\
\hline \multirow[t]{3}{*}{ Disociación Fantasía } & Normal & 111 & $(68,5)$ & 98,03 & 2 &, 00 \\
\hline & Riesgo & 40 & $(24,7)$ & & & \\
\hline & Clínico & 11 & $(6,8)$ & & & \\
\hline
\end{tabular}


Tabla 3

Frecuencia del uso de las estrategias de afrontamiento de la población participante

\begin{tabular}{|c|c|c|c|c|c|c|}
\hline Estrategia de afrontamiento & & $\mathbf{F}$ & $(\%)$ & Chi-cuadrado & GI & $\mathbf{P}$ \\
\hline \multirow[t]{2}{*}{ Olvidar } & $\mathrm{Si}$ & 122 & $(75,3)$ & 41,50 & 1 &, 00 \\
\hline & No & 40 & $(24,7)$ & & & \\
\hline \multirow[t]{2}{*}{ Jugar } & $\mathrm{Si}$ & 112 & $(69,1)$ & 23,72 & 1 &, 00 \\
\hline & No & 50 & $(30,9)$ & & & \\
\hline \multirow[t]{2}{*}{ Permanecer solo } & $\mathrm{Si}$ & 41 & $(25,3)$ & 39,50 & 1 &, 00 \\
\hline & No & 121 & $(74,7)$ & & & \\
\hline \multirow[t]{2}{*}{ Permanecer callado } & $\mathrm{Si}$ & 56 & $(34,6)$ & 15,43 & 1 &, 00 \\
\hline & No & 106 & $(65,4)$ & & & \\
\hline \multirow[t]{2}{*}{ Ver el lado bueno de las cosas } & $\mathrm{Si}$ & 134 & $(82,7)$ & 69,35 & 1 &, 00 \\
\hline & No & 28 & $(17,3)$ & & & \\
\hline \multirow[t]{2}{*}{ Culparse } & $\mathrm{Si}$ & 54 & $(33,3)$ & 18,00 & 1 &, 00 \\
\hline & No & 108 & $(66,7)$ & & & \\
\hline \multirow[t]{2}{*}{ Culpar a otro } & $\mathrm{Si}$ & 26 & $(16,0)$ & 74,69 & 1 &, 00 \\
\hline & No & 136 & $(84,0)$ & & & \\
\hline \multirow[t]{2}{*}{ Dejar que las cosas se arreglen solas } & $\mathrm{Si}$ & 149 & $(92,0)$ & 114,17 & 1 &, 00 \\
\hline & No & 13 & $(8,0)$ & & & \\
\hline \multirow[t]{2}{*}{ Hacer algo para arreglar las cosas } & $\mathrm{Si}$ & 139 & $(85,8)$ & 83,06 & 1 &, 00 \\
\hline & No & 23 & $(14,2)$ & & & \\
\hline \multirow[t]{2}{*}{ Gritar } & $\mathrm{Si}$ & 26 & $(16,0)$ & 74,69 & 1 &, 00 \\
\hline & No & 136 & $(84,0)$ & & & \\
\hline \multirow[t]{2}{*}{ Calmarse } & $\mathrm{Si}$ & 147 & $(90,7)$ & 107,55 & 1 &, 00 \\
\hline & No & 15 & $(9,3)$ & & & \\
\hline \multirow[t]{2}{*}{ Desear algo } & $\mathrm{Si}$ & 139 & $(85,8)$ & 83,06 & 1 &, 00 \\
\hline & No & 23 & $(14,2)$ & & & \\
\hline \multirow[t]{2}{*}{ Hacer algo diferente } & $\mathrm{Si}$ & 143 & $(88,3)$ & 94,91 & 1 &, 00 \\
\hline & No & 19 & $(11,7)$ & & & \\
\hline \multirow[t]{2}{*}{ Intentar sentirme mejor } & $\mathrm{Si}$ & 141 & $(87,0)$ & 88,88 & 1 &, 00 \\
\hline & No & 21 & $(13,0)$ & & & \\
\hline \multirow[t]{2}{*}{ No hacer nada } & $\mathrm{Si}$ & 67 & $(41,4)$ & 4,84 & 1 &, 02 \\
\hline & No & 95 & $(58,6)$ & & & \\
\hline
\end{tabular}

Tabla 4

Datos Descriptivos de resiliencia

\begin{tabular}{lcccccc}
\hline \multicolumn{1}{c}{ Resiliencia } & MEDIA & (D.E) & MÍNIMO & MÁXIMO & I.C.L.I & I.C.L.S \\
\hline Total & 110,24 & 21,31 & 27,00 & 135,00 & 106,79 & 113,69 \\
Autoestima & 35,41 & 7,72 & 9,00 & 45,00 & 34,16 & 36,66 \\
Redes & 37,97 & 7,67 & 9,00 & 45,00 & 36,73 & 39,22 \\
Aprendizaje & 36,84 & 7,04 & 9,00 & 45,00 & 35,70 & 37,98 \\
Recursos internos & 53,03 & 10,48 & 13,00 & 65,00 & 51,33 & 54,73 \\
Recursos externos & 33,18 & 7,03 & 8,00 & 40,00 & 32,04 & 34,32 \\
\hline
\end{tabular}


Análisis de la percepción de atención en saludy necesidad de atención en la población infantil y adolescente

Este análisis muestra que en general en este grupo poblacional existe una percepción positiva de la atención en salud $(1,73 / 2,56)$, pero al mismo tiempo hay una alta necesidad manifiesta de esta atención y de que mejore $(3,71 / 3,85)$.

\section{Factores de riesgo relacionados con las problemáticas psicológicas}

El análisis de factores de riesgo se hizo a través de la obtención de la razón de ventajas para las variables de edad, sexo, estudio y grado de escolaridad en relación con las características sintomáticas evaluadas por el CBCL y elYSR.

El análisis de resultados muestra que el ser de sexo masculino es un factor de riesgo para la presentación de depresión ( $\mathrm{rv}=3,42$; IC: 95\% Li: 1,57 Ls: 7,43), problemas sociales ( $\mathrm{rv}=3,79 ; \mathrm{IC}: 95 \% ; \mathrm{Li}=1,56 ; \mathrm{Ls}: 9,18)$ y agresión (rv $=2,73 ; \mathrm{IC}: 95 \%$; Li=1,23; Ls: 6,02) en el grupo de niños. A efectos de la obtención de la razón de ventajas, tanto la depresión, como los problemas sociales y la agresión se dicotomizaron, agrupando las categorías de riesgo y clínica del CBCL en una sola y dejando la clasificación de normal como otra categoría.

En el grupo de adolescentes, ser de sexo masculino es un factor de riesgo para agresión (rv: 1,38; IC: 95\%; Ls: $6,62 ; \mathrm{Li}: 1,23)$, mientras que la edad hasta 12 años $(\mathrm{rv}=2,13$ IC: $95 \% ; \mathrm{li}=1.06 ; \mathrm{Ls}=4,28$ ) y el menor grado educativo, hasta haber cursado estudios de primaria (rv $=2,37 \mathrm{IC}: 95 \%$; $\mathrm{Li}=1,07 ; \mathrm{Ls}=5,25)$, son factores de riesgo para el desarrollo de problemas somáticos. De nuevo, para este análisis, se obtuvo una sola categoría con las clasificaciones de riesgo y clínica del CBCL y otra con la de normal.

\section{DISCUSIÓN}

El objetivo de la presente investigación fue caracterizar los síntomas psicológicos de la población infantil y adolescente expuesta al conflicto armado en una zona rural de Colombia. Se evidencia en el $49 \%$ de los niños y en el $80 \%$ de los adolescentes expuestos a hechos victimizantes en circunstancias de conflicto armado, la presencia de conductas externalizadas, y en el $70 \%$ de los niños y en el $83 \%$ de los adolescentes, conductas internalizadas de depresión y ansiedad, en los niveles de afectación de rango clínico y de riesgo. En el $88 \%$ del grupo de adolescentes prevalecen los problemas de pensamiento en rango clínico y de riesgo, y en el $44 \%$ de los niños es significativa la presencia de los problemas somáticos en dichos rangos. En los adolescentes se identifica mayor presencia de rompimiento de normas (58\%), mientras que en los niños se observan conductas agresivas (33\%).
Estos resultados son acordes con lo señalado por diferentes autores (Betancourt et al., 2012; Haroz et al., 2013; Mels, 2012; Hasanovic, 2011; Layne et al., 2010; Posada et al., 2011), quienes también evidenciaron en sus investigaciones que los niños y adolescentes expuestos a situaciones de violencia generan conductas internalizadas y externalizadas. La presencia de problemas de pensamiento en los adolescentes está relacionada con la etapa de desarrollo cognitivo en la que se encuentran (Cohen \& Mannarino, 2008; López- Navarrete et al., 2007; Pereda, 2012). Los niños en esta etapa del desarrollo pueden diferir ampliamente en las interpretaciones que hagan de las situaciones dependiendo de su nivel de abstracción. La guerra lleva a un cambio en el estilo de vida y a la pérdida de seres queridos, lo que puede incrementar la presencia de los problemas de pensamiento y somatización (Fremont, 2004).

Con respecto a la presencia de conductas externalizadas, las investigaciones han señalado que durante la etapa escolar se hacen más evidentes los comportamientos agresivos, irritabilidad, rabietas, conductas oposicionistas y destructivas, que ligadas a la exposición a eventos violentos, aumentan su aparición (Pereda, 2012). En una población infantil y adolescente expuesta a situaciones de guerra posiblemente se incrementan estos comportamientos. El contexto en que se encuentran ubicados, caracterizado por deficiencia económica, ruptura familiar, exclusión social, inestabilidad e inseguridad, afecta las condiciones diarias en las que se desarrollan y causa aflicciones adicionales y problemas externalizados (Mels, 2012).

Los puntajes en las escalas de depresión y ansiedad denotan que el $70 \%$ de los niños y el $60 \%$ de los adolescentes evaluados se encuentran en un nivel de afectación de riesgo y clínico. Esto es producto de la exposición a hechos victimizantes como lo señalan los estudios sobre el impacto de la guerra en niños y niñas, los cuales han demostrado consecuencias como déficits cognoscitivos, limitaciones en la comunicación funcional, mayor probabilidad de tener respuestas agresivas ante situaciones violentas, comportamientos de ansiedad y depresión, sintomatología postraumática, actitudes desafiantes - belicosas y comportamientos delictivos (Boothby, 2008; Gutiérrez \& Acosta 2013; López-Navarrete et al., 2007; Pereda, 2012).

Llama la atención que el $55 \%$ de la población adolescente se encuentra en riesgo de presentar un TEPT, mientras que el $6 \%$ lo presenta actualmente. Estos resultados son similares a los encontrados en estudios realizados usando evaluaciones estructuradas válidas estadísticamente, los que han mostrado que las tasas de TEPT en niños expuestos a las actividades terroristas varían del 28 al 50\% (Fremont, 2004). Sin embargo, se encuentran por encima de los encontrados en el primer estudio de salud mental del adolescente realizado 
en Colombia (Ministerio de Protección Social, Universidad CES \& la Oficina de Naciones Unidas contra la Droga y el Delito-[UNODC], 2010), que muestran una prevalencia de vida para este TEPT del 1.4\%, y cuyos datos son inferiores a los encontrados en otros estudios realizados con niños colombianos expuestos a la guerra, que arrojan un $16.8 \%$ y un $23.2 \%$ de prevalencia de dicho trastorno en niños de dos municipios sometidos a situaciones de hostigamiento armado (Calderón \& Barrera 2012). Al analizar la población en riesgo de presentarlo, el porcentaje es superior a los datos hallados en dicho estudio (Calderón \& Barrera, 2012). Es de destacar que en el grupo poblacional de esta investigación se encuentran altos niveles de resiliencia y adecuadas habilidades de afrontamiento, lo que puede explicar la baja presencia de estrés postraumático en nivel clínico. Adicionalmente, el $76 \%$ de estos niños estaba bajo el cuidado y protección de sus padres en el tiempo de la exposición al conflicto armado, circunstancia que según los estudios previene la presencia de TEPT (Masten \& Narayan, 2012).

De otra parte, a pesar de que el impacto psicológico del terrorismo en los menores es significativo y lleva a respuestas emocionales de ira (Bell et al., 2012), en esta investigación no se evidenciaron niveles clínicos en el grupo de adolescentes, el $87 \%$ de los evaluados no la presentan. Muy seguramente los niveles de resiliencia, las habilidades de afrontamiento y las redes de apoyo con que cuenta este grupo poblacional sirven como factores protectores que llevan a regular esta respuesta emocional. Igualmente, los resultados concuerdan con un estudio realizado en Sri Lanka, el cual concluye que un estilo de vida que involucre la espiritualidad, sólidas relaciones familiares y comunitarias, permite contrarrestar los efectos negativos de la guerra sobre los niños (Fernando \& Ferrari, 2011).

Adicionalmente, los resultados del TSCC evidencian que en el $39 \%$ de la población de estudio hay riesgo de disociación, y el 57\% de la población no presenta problemas. Estos resultados permiten señalar un efecto significativo de la guerra en la salud mental de este grupo de participantes. Sin embargo, el porcentaje de población normal (57\%), lleva a considerar que a pesar de la situación traumática por la que han pasado, ciertas características individuales, en este caso la resiliencia y las habilidades de afrontamiento, posiblemente hacen que estos adolescentes se sobrepongan a los efectos de la guerra y, por ende, logren un mejor ajuste social y comportamental.

Con respecto al consumo de alcohol, los resultados del CAGE evidenciaron que el $1 \%$ de la población adolescente se encuentra en consumo perjudicial y un $6 \%$ en consumo de riesgo; no obstante, se observa un alto porcentaje en consumo moderado (93\%) en una población joven como son los adolescentes. Probablemente este comportamiento se relacione con la presencia del $59 \%$ de conductas externalizadas que se encuentran en el YSR en nivel clínico, lo que muestra consonancia con diversos trabajos en los que se enuncia que los niños y niñas adolescentes presentan conductas externalizadas como el uso del alcohol para afrontar el efecto inmediato del terrorismo (Pereda, 2012).

De otro lado, se observa que este grupo poblacional utiliza como principales estrategias de afrontamiento, dejar que la situación se arregle por sí sola, arreglar haciendo olvidar, usar el lado bueno de las situaciones, no culpabilizarse, ni culpar a otros, no usar los gritos, calmarse, hacer algo diferente e intentar sentirse mejor. Dichas estrategias evidencian que el afrontamiento cognitivo y conductual se centra en la distracción, reestructuración cognitiva, resolución de problemas y regulación emocional, lo que es reflejo de funcionalidad en dichas habilidades. El uso de estas estrategias supone un esfuerzo cognitivo por valorar y destacar los aspectos positivos del problema, poniendo en marcha acciones efectivas que tiendan a solucionar las situaciones que les resultan conflictivas, lo que facilita su desarrollo social. Aunque pocos estudios han analizado específicamente las estrategias de afrontamiento utilizadas por los menores luego de un atentado terrorista, se indica que éstos utilizan diversas formas, tanto las centradas en la gestión de las emociones provocadas por el terror como las estrategias más centradas en el problema, siendo la percepción de control que el menor tenga de la situación, una de las variables que se ha relacionado con mayor respuesta adaptativa (Pereda, 2012).

Con respecto a los factores de riesgo en esta población, en el grupo de niños el ser hombre menor de doce años y hallarse en una zona de postconflicto, aumenta la probabilidad de presentar depresión, agresión y problemas sociales, lo cual es coherente con hallazgos que denotan la prevalencia de conductas externalizadas en esta población (Pereda, 2012). Estos resultados muestran las afectaciones psicológicas que se generan en los niños en zonas de conflicto armado, que al no ser intervenidas, pueden convertirse en patologías que perduran hasta la edad adulta.

En los adolescentes se identifican como factores de riesgo los problemas de agresión y somáticos, el menor grado escolar, la edad y el sexo masculino. Los datos obtenidos concuerdan con otra investigación en la que se observa que la exposición a la violencia y a los estresores diarios varía según sexo y edad, los adolescentes varones están más expuestos que las adolescentes mujeres, y a su vez, ambos en el grupo de mayor edad, están más expuestos que los integrantes del grupo de menor edad (Mels, 2012). Si bien estas diferencias no se han encontrado en todos los estudios, se ha analizado la gravedad de la sintomatología 
postraumática presente en menores. Las niñas muestran más síntomas que los niños, pero los que ellos presentan son de mayor gravedad (Pereda, 2012), lo que corrobora que la exposición a la violencia armada tiene una afectación significativa en ambos géneros.

La investigación realizada permitió corroborar que evaluar una población que ha sido expuesta a la guerra, conlleva limitaciones relacionadas con la posible re-victimización, el temor de la población, el bloqueo emocional y el tipo de instrumentos a utilizar entre otras. Sus resultados reflejan la afectación psicológica significativa de la salud mental en niños y adolescentes expuestos a la violencia producto del conflicto armado en Colombia, lo que señala la urgencia de definir, desarrollar y evaluar programas de atención psicosocial e intervención clínica orientados a mitigar el impacto, a prevenir problemas de salud mental, y a favorecer el ajuste emocional y la calidad de vida de la población víctima del conflicto armado.

\section{REFERENCIAS}

Achenbach, T. y Rescorla, L. (2001). Manual for the ASEBA School: Age Forms \& Profiles. Child Behavior Checklist for ages 6-18, Teacher's Report Form youth self-report an Integrated System of Multi - Informant. Assessment. Burlington: University of Vermont.

Al-Obaidi, A., Budosan, B., \& Jeffrey, L. (2010). Child and Adolescent Mental Health in Iraq: current situation and scope for promotion of child and adolescent mental health policy. Journal Intervention, 8(1), 40 - 51.

Alzate, M. L. (2012). Acciones colectivas frente a la violencia. Disquisiciones a partir de un estudio de caso: Comuna 13 de Medellín (Colombia). Revista Forum, 2(3), 111-130.

Bastidas, W., \& Rodríguez, A. I. (2010). Víctimas en Medellín. El Ágora Universidad de San Buenaventura, 10(2), 367-397.

Bell, V., Méndez, F., Martínez, C., Palma, P. P., \& Bosch, M. (2012). Characteristics of the Colombian armed conflict and the mental health of civilians living in active conflict zones. Journal Conflict and Health, 6(1), 1-8.

Betancourt, T. S., Borisova, I., Williams, T. P., Meyers-Ohki, S. E., Rubin-Smith, J. E., Annan, J., \& Kohrt, B. A. (2013). Research review: Psychosocial adjustment and mental health in former child soldiers-A systematic review of the literature and recommendations for future research. Journal of Child Psychology and Psychiatry, 54(1), 17-36.

Betancourt, T. S., \& Khan, K. T. (2008). The mental health of children affected by armed conflict: protective processes and pathways to resilience. International Review of Psychiatry, 20(3), 317-328.

Betancourt, T. S., McBain, R., Newnham, E. A., \& Brennan, R. T. (2012). Trajectories of Internalizing Problems in WarAffected Sierra Leonean Youth: Examining Conflict and Postconflict Factors. Journal Child Development, 84(2), 455-470.
Boothby, N. (2008). Political violence and development: an ecologic approach to children in war zones. Child and Adolescent Psychiatric Clinics of North America, 17(3), 497514.

Briere, J. (1996) Trauma Symptom Checklist for Children: Professional manual. Florida: Psychological Assessment Resources Inc.

Calderón, L., \& Barrera, M. (2012). Exploración neuropsicológica de la atención y la memoria en niños y adolescentes víctimas de la violencia en Colombia: estudio preliminar. Revista CES Psicología, 5(1), 39-48.

Cohen, J. A., \& Mannarino, A. P. (2008). Trauma-Focused Cognitive Behavioural Therapy for Children and Parents. Journal Child and Adolescent Mental Health, 13, (4), 158-162.

Comer, J. S., \& Kendall, P. C. (2007). Terrorism: The psychological impact on youth. Journal Clinical Psychology: Science and Practice, 14(3), 179-212.

Cuevas, M.C (2006). Exposición a violencia, conductas parentales y afrontamiento en niños y adolescentes. Tesis doctoral. Universidad de Granada.

Fazel, M., Reed, R. V., Panter-Brick, C., \& Stein, A. (2012). Mental health of displaced and refugee children resettled in high-income countries: risk and protective factors. The Lancet, 379(9812), 266-282.

Feldman, R., \& Vengrober, A. (2011). Posttraumatic stress disorder in infants and young children exposed to warrelated trauma. Journal of the American Academy of Child \& Adolescent Psychiatry, 50(7), 645-658.

Fernando, C., \& Ferrari, M. (2011).Spirituality and Resilience in Children of War in Sri Lanka. Journal of Spirituality in Mental Health, 13, (1), 52-77.

Fremont. W., P. (2004). Childhood reactions to terrorisminduced trauma: a review of the past 10 years. Journal of American Academy of Child and Adolescent Psychiatry, 43(4), 381- 392.

Gutiérrez, I., \& Acosta, A. (2013). La violencia contra niños y niñas: un problema global de abordaje local, mediante la IAP. Revista Latinoamericana de Ciencias Sociales, Niñez y Juventud, 11(1), 261-272.

Haroz, E. E., Murray, L. K., Bolton, P., Betancourt, T., \& Bass, J. K. (2013). Adolescent Resilience in Northern Uganda: The Role of Social Support and Prosocial Behavior in Reducing Mental Health Problems. Journal of Research on Adolescence, 23(1), 138-148.

Hasanović, M. (2011). Psychological consequences of wartraumatized children and adolescents in Bosnia and Herzegovina. Acta Médica Académica, 40(1), 45-66.

Hewitt, N., Jaimes, S., Vera., L.A., y Villa, M.C. (2012). Características psicométricas del cuestionario de comportamientos infantiles CBCL en niños y adolescentes colombianos. Trabajo de Grado. Facultad de Psicología. Universidad de San Buenaventura, Bogotá - Colombia. 
Hobfoll, S. E., Mancini, A. D., Hall, B. J., Canetti, D., \& Bonanno, G. A. (2011). The limits of resilience: Distress following chronic political violence among Palestinians. Social Science \& Medicine, 72(8), 1400-1408.

Layne, C. M., Olsen, J. A., Baker, A., Legerski, J. P., Isakson, B., Pašalić, A.,\& Pynoos, R. S. (2010). Unpacking trauma exposure risk factors and differential pathways of influence: predicting postwar mental distress in Bosnian adolescents. Child development, 81(4), 1053-1076.

Londoño, N. H., Patiño, C. D., Restrepo, D., Correa, J. Raigoza, J., Toro, L., Jaramillo, G \& Rojas, C. (2008) Perfil Cognitivo Asociado al Trastorno por Estrés Postraumático en Víctimas de Violencia Armada. Informes Psicológicos, 10(11), 27.

López-Navarrete, G. E., Perea-Martínez, A., Loredo, A. A., Trejo-Hernández, J., \& Jordán-González, N. (2007). Niños en situación de guerra. Acta Pediátrica Mexicana, 28(2), $74-80$

Masten, A. S., \& Narayan, A. J. (2012). Child development in the context of disaster, war, and terrorism: Pathways of risk and resilience. Annual review of psychology, 63, 227-257.

Mels, C. (2012). Entre pobreza y violencia: ¿cómo afectan los estresores diarios y la violencia a adolescentes en zonas de guerra? Ciencias Psicológicas, 6(2), 111-122.

Mels, C., Derluyn, I., Broekaert, E., \& Rosseel, Y. (2009). Screening for traumatic exposure and posttraumatic stress symptoms in adolescents in the war-affected Eastern Democratic Republic of Congo. Archives of Pediatrics and Adolescent Medicine, 163(6), 525 - 530.

Miller, K. E., \& Rasmussen, A. (2010). War exposure, daily stressors, and mental health in conflict and post-conflict settings: bridging the divide between trauma-focused and psychosocial frameworks. Journal Social Science \& Medicine, 70(1), 7-16.

Ministerio de Protección Social, Universidad CES y la Oficina de Naciones Unidas contra la Droga y el Delito -UNODC (2010). Situación de la salud mental del adolescente, Estudio Nacional de la Salud Mental Colombia. Bogotá: Ministerio de protección social.

Murthy, R. S., \& Lakshminarayana, R. (2006). Mental health consequences of war: a brief review of research findings. World Psychiatry, 5(1), 25 - 30.

Neuner, F., Catani, C., Ruf, M., Schauer, E., Schauer, M., \& Elbert, T. (2008). Narrative exposure therapy for the treatment of traumatized children and adolescents (KidNET): from neurocognitive theory to field intervention. Journal Child and adolescent psychiatric clinics of North America, 17(3), 641-664.

Organización Mundial de la Salud. (2012). Adolescent health. Recuperado de. http://www.who.int/topics/adolescent_ health/en/index.html

Pereda, N. (2012). Menores Víctimas del Terrorismo: Una Aproximación desde la Victimología del Desarrollo. Anuario de Psicología Jurídica, 22, 13-24.
Pérez-Olmos, I., Fernández-Piñeres, P. E., \& Rodado-Fuentes, S. (2005). Prevalencia del trastorno por estrés postraumático por la guerra, en niños de Cundinamarca, Colombia. Revista de salud pública, 7(3), 268-280.

Pine, D. S., \& Cohen, J. A. (2002).Trauma in children and adolescents: Risk and treatment of psychiatric sequelae. Biological Psychiatry, 51(7), 519-531.

Posada Gilède, R., \& Parales Quenza, C. J. (2011). Violencia y desarrollo social: más allá de una perspectiva de trauma. Universitas Psychological, 11(1), 255-267.

Rescorla, L., Ivanova, M., Achenbach, T., Begovac, I., Chahed, M., Drugli, M.B., Ribas, D., Fung, D.S., Haider, M., Hansson, K., Hewitt, N., ᄀLarsson, B., Maggiolini, A., Markovic, J., Mitrovic, D., Moreira, P., Oliveira, J.T., Olsson, M., Phaik Ooi, Y., Villa, M.C., Petot, D., Pisa, C., Pomalima, R., Monzani, M., Rudan, V., Sekulic, S., Shahini, M., Silvares, E., Szirovicza, L., Jaimes, S., Valverde, J., Vera, L.A., Viola, L., y Woo, B.S.C., (2012). International Epidemiology of Child and Adolescent Psychopathology: 2.Integration and Applications of Dimensional Findings from 44 Societies. Journal of the American Academy of Child and Adolescent Psychiatry, 51(12), 1273-1283.e8.

Rieder, M., \& Choonara, I. (2012). Armed conflict and child health. Archives of Disease in Childhood, 97(1), 59-62.

Saavedra, E. y Villalta, M. (2008). Escala de Resiliencia SVRES, para jóvenes y adultos. Segunda Edición. Santiago: autores.

Sanchez-Padilla, E., Casas, G., Grais, R. F., Hustache, S., \& Moro, M. R. (2009). The Colombian Conflict: A Description of a Mental Health Program in the Department of Tolima. Journal Conflict and Health, 3(13), 1 - 6.

Spirito, A., Stark, L. J. \& Williams, C. (1988). Development of a brief coping checklist for use with pediatric populations. Journal of Pediatric Psychology, 13(4), 555-574.

Tamashiro, T. (2010). Impact of Conflict on Children's Health and Disability. "Paper commissioned for the Education for All Global Monitoring Report - EFA Global Monitoring Report 2011. The hidden crisis: Armed conflict and education". Pp. 1-14.

Tol, W. A., Song, S., \& Jordans, M. J. (2013). Annual Research Review: Resilience and mental health in children and adolescents living in areas of armed conflict-a systematic review of findings in low-and middle-income countries. Journal of Child Psychology and Psychiatry, 54(4), 445-460. doi: 10.1111/jcpp.12053

Yule, W., Dyregrov, A., Raundalen, M., \& Smith, P. (2013). Children and war: the work of the Children and War Foundation. European Journal of Psychotraumatology, 4, 18424

Ziaian, T., de Anstiss, H., Antoniou, G., Baghurst, P., \& Sawyer, M. (2012). Resilience and its Association with Depression, Emotional and Behavioural Problems, and Mental Health Service Utilisation among Refugee Adolescents Living in South Australia. International Journal of Population Research, 1-9. doi:10.1155/2012/485956. 